\title{
Aerztliches Unterstützungs- und Versicherungswesen in
} Deutschland.

Von J. Schwalbe.

Das deutsche ärztliche Unterstützungs- und Versicherungswesen hat im verflossenen Jahre nannigfache Erweiterungen erfahren, die so gering sie auch an sich sein mögen - freudig anerkannt werden müssen. Bei der socialen Nothlage, in der sich der ärztliche Stand Deutschlands aus den sattsam erörterton Gründen seit Jahren befindet, verdient jeder Versuch der Collegen zur Selbsthülfe, sei es um den in Drangsal gerathenen Aerzten oder deren Hinterbliebenen eine standeswürdige Existenz zu ermöglichen, sei es - was unendlich viel höher geschätzt werden muss - um sich bezw. die Angehörigen gegen eine aus Krankheit und Tod erwachsende Misere zu schützen, unseren voll: sten Beifall. Nicht oft und eindringlich genug kann darauf hingewiesen werden, dass Niemand vor den Wechselfällen des Lebens sicher ist und dass Jeder nach bestèn Kräften bei Zeiten sein Haus bestelle. Die schlimmen Folgen, die eine Missachtung dieser Lehre nach sich ziehen kann, zeigen die traurigen Beobachtungen, die wir nicht selten in der 
Collegenschaft zn machen Gelegenheit haben, das beweisen namentlich die Bittgesuche, die zn Dutzenden jahrans jahrein bei den ärztlichen Unterstïtzlungskassen eingereicht werden. Der letzte Rechenschaftsbericht, den der Aerztliche Central-Anzeiger (No. 47) iiber seine „Wittwengabe" veröffentlicht, theilt uns mit, dass in den ersten 3 Quartalen des Jahres 1898 die Bittgesuche von 125 Wittwen und Waisen verstorbener Collegen, welche nach genauester Prüfung ihrer Verhältnisse anf die Empfehlıng zweier Collegen hin als würdig und bedürftig erkannt wurden, u nber ücksichtigt bleiben mussten! Wie gross muss die Noth derjenigen sein, die sich gezwungen fühlen, zweien Collegen ihre tranrige Lage zll entdecken und sie um ihre Hülfe zur Erlangung eines Almosens von $60 \mathrm{M}$. bezw. $30 \mathrm{M}$. pro Quartal anzugehen! Und wie bedanernswerth diejenigen, die selbst einer so geringen Gabe ans Mangel an vorhandenen Mitteln nicht theilhaftig werden! - Solchen Thatsachen und Erwägungen gegenüber wollen wir nicht ermïden, die vorbengende Fürsorge der Collegen für sich selbst und ihre Nächstenliebe für die bedürftigen Standesgenossen immer wieder und wieder anzurufen.

Die Wege, auf denen ein Ausbau des deutschen ärztlichen Unterstiitzungs- ind Versichernngswesens geschehen kann, hat die im Jahre 1896 veröffentlichte Denkschrift der Berlin-Brandenburger Aerztekammer eingehend nachgewiesen. Und mit Genugthuung kann festgestellt werden, dass der Appell, den sie an Dentschlands Aerzte gerichtet hat, nicht ungehört verhallt ist, dass die Saatkörner, die nnsere Kammer ansgestrent hat, in manchen Theilen unseres Vaterlandes aufgegangen sind. Wie aus privaten Mittheilungen, die mir zugegangen sind, und ans verschiedenen Veröffentlichungen erhellt, sind einige der Institutionen, ïber die ich in den folgenden Zeilen berichten will, durch die Denkschrift der Berlin-Brandenburger Aerztekammer ins Leben gernfen und theilweise sogar anf die dort niedergelegten Thesen anfgebaut worden.

Die geringsten Erfolge sind fïr Prenssen zu verzeichnen. Nicht etwa, weil anch hier es sich bewahrheitet hätte, dass der Prophet im eigenen Vaterlande nichts gilt - die Grundzüge der Denkschrift sind fast von allen Aerztekammern richtig gewürdigt und anerkannt worden sondern weil jede Initiative durch das in Anssicht stehende Gesetz über das Umlagerecht der Aerztekammern gehemmt wurde. Durch dieses Gesetz, dessen Vorlage in der nächsten Landtagssession zn erwarten ist, wird zwar lediglich das Unterstütznngswesen berïhrt, allein man hat sich auch für das Versicherungswesen mit gebunden gefühlt, für welches ja ohnehin Verständniss und Neigung im Allgemeinen weniger entwickelt ist. Man blickt anf die grossen Versicherungssummen und auf die verschiedenen Versicherungsarten nnd scheut vor der Schwierigkeit der ganzen zu lösenden Aufgaben zuriïck: da man der Meinung ist, dass die Aerzte sich nicht zur Versicherung gegen Krankheit, Invalidität und Tod entschliessen werden, steht man von der Einrichtung jeglicher Kasse ab, statt es wenigstens mit der Gründung einer einzigen der 3 Kategorieen zu versuchen. Durch den ständigen Blick auf das Ganze geht der Vortheil des Einzelnen verloren.

Dabei ist die Möglichhkeit, eine der genannten Versicherungen abzuschliessen, für die deutschen Aerzte in ausgezeichnetem Maasse vor* handen. Die bisherigen Vorziige der Centralhilfskasse oder, wie sie seit dem vorigen Jahre heisst, der „Versicherungskasse für die A erzte Deutschlands (früher Centralhilfskasse)“ sind so oft dargelegt und anerkannt worden, dass wir von einer Schilderung und Empfehlung ihrer Organisation an dieser Stelle absehen können. Dagegen verdient es als ein grosser Fortschritt hervorgehoben zu werden, dass die Versicherungskasse im verflossenen Jahre $11 \mathrm{~m}$ zwei wichtige Abtheilungen vermehrt worden ist, die sich zweifellos die Sympathieen der Aerzte erwerben werden, nämlich um eine Sterbekasse und eine Wittwenkasse; ganz besonders mit der Schöpfung der letzteren ist ein wiederholt ansgesprochener Wunsch der Aerzte erfüllt worden. Was die wesentlichsten Grundlagen der beiden Kassen betrifft, so gelten für die Aufnahme in die Sterbekasse, die Beitragsleistıngen, Rechte und Pflichten der Mitglieder die allgemeinen Bestimmungen der Centralhilfskasse und ein eigener Tarif (S). Besonders zu bemerken ist, dass nicht nur der einzelne Arzt, sondern anch ein ganzer Verein in corpore aufgenommen werden kann. Während aber der Einzelne olıne ärztliches Zengniss, lediglich auf die Bestätignung des Inhalts des Aufnahmeantrags durch zwei den Antragsteller kennende Aerzte zugelassen wird, muss von einem Verein für den Beitritt beigebracht werden: 1. eine Urkunde über den Beschluss einer obligatorischen Sterbekassenversicherung der gesammten Vereinsmitglieder bei der Versichernungskasse der Aerzte Dentschlands, 2. eine vom Vorstand des Tereins beglaubigte Liste über Zahl, Name, Geburtstag und- jahr der dem Verein mindestens ein Jahr angehörenden Mitglieder. - Die Prämie der versicherten Vereine wird entsprechend dem Durchschnittsalter und der Zahl der Mitglieder der gesammten Vereine für jedes Jahr nen berechnet and ist in einmaliger jährlicher Gesammtzahlung von dem Vereinsvorstande zul entrichten. Der Verein erhält fïr seine Mitglieder einen alljährlich zu ernevernden, die Namen der versicherten Mitglieder auffuhrenden Gesammtmitgliedschein.

Aus dem Tarif der Sterbekasse heben wir heraus, dass fïr $500 \mathrm{M}$. Sterbegeld die Vierteljahrsprämie bei einem Eintrittsalter von 30 Jahren 2,95 M., bei einem Eintrittsalter von 60 Jahren 9,70 M. beträgt.
Die Bedenken über die Lebensfähigkeit der Wittwenkasse sind von vornherein dadurch beseitigt, dass eine Rückversicherung der Mitglieder bei der gnt fundirten Hinterbliebenen-Kasse des Verbandes deutscher Beamtenvereine eingerichtet ist. Auf die einzelnen Satzıngen der Wittwenkasse können wir hier nicht eingehen. Als besondere Vorzüge der Organisation seien nur hervorgehoben: 1. dass keine eigentliche Karenzzeit besteht, sondern dass die volle Rente bereits nach 3 jähriger, $4 / 6$ der Rente nach 2 jähriger, $2 \%$ nach 1 jähriger Versicherungsdauer gewährt wird, und dass nach weniger als 1 jähriger Versicherungsdauer die eingezahlte Prämie zurückgezahlt wird; 2 . dass ausser dem einfachen Tarif noch ein solcher mit Rückgewähr der eingezahlten Prämien besteht fïr den Fall, dass die Fran des Versicherten vor ihm stirbt; 3. dass anch höhere Altersstufen, bis zum 65. Lebensjahre, zm! Versichermng zugelassen werden. - Aus dem Tarif der Wittwenkasse (mit Dividendengenuss) ist zu nennen: die vierteljährliche Prämie für eine jährliche Wittwenpension von $60 \mathrm{M}$. beträgt, wenn das Mitglied beim Eintritt $30 \mathrm{Jahre}$ und die Frau $20 \mathrm{Jahre}$ alt ist, 3,80 M., dasselbe bei Rïckgewähr der Prämien 4,70 M.

Ausser diesen wichtigen Erweiterungen der Centralhilfskasse sind im Jahre 1898 noch eine Reihe bemerkenswerther Neuerungen in den Satzungen getroffen worden; als wesentlich sind aufzuführen: die Einfülırung neuer Tarife, welche die Rückzahlung der gesammten gezahlten Prämien gewähren, fïr den Fall, wo der Tod des Versicherten eintritt, ehe derselbe in den Genuss der versicherten Rente gelangte, und ferner ein neuer niedrigerer Tarif für die Alterspensionskasse. - Durch alle diese Erweiterungen, die zumeist ihrem durch hervorragende Sacllkenntniss und rastlosen Eifer ausgezeichneten Obmann, Collegen Bensch, zu verdanken sind, ist die Centralhilfskasse sehr erheblich vervollkommnet worden, und es ist nur zu wünschen, dass die Collegen in grősserem Maasse als bisher von den Einrichtungen dieser Institution Gebranch machen möchten.

Ansser diesem Ausbau in der Organisation der „Versicherungskasse für die Aerzte Dentschlands“ ist alf dem Gebiete des preussischen Unterstiitzanngs- nnd Versicherungswesens aus dem letzten Jahre kein Fortschritt zil vermelden. Dagegen möchte ich der Vollständigkeit und des allgemeinen Interesses halber eine Reihe preussischer Kassen anführen, die in meiner Uebersicht vom Jahre $1895^{1}$ ) fehlen, weil sie damals nicht zu meiner Kenntniss gelangt, beziehungsweise auch erst später gegründet worden sind. Es sind:

1. Provinz Westpreussen: Sterbekasse des Vereins der Bahnärzte der Kgl. Direktionen Bromberg, Danzig, Königsberg (Sitz in Danzig, gegründet 1891, obligatorischer Beitritt der zuständigen Bahnärzte; nnterstützt nur Mitglieder; Jahresbeitrag $5 \mathrm{M}$., Sterbegeld 300 M., Gesammtsinmme der bisher gezahlten Sterbegelder 5100 M., Mitgliederzahl im Jahre 1898:181, Vereinsvermögen 2990 M., Sterbegelder im Jahre 1897 für 6 Todesfälle 1800 M.). Berlin.

2. die Unterstützungskasse des ärztlichen Nordclub in

3. Provinz Brandenburg: die Unterstütungskasse des Vereins der Aerzte der Niederlausitz (Sitz in Cottbus, gegründet 1887, obligatorischer Beitritt der Vereinsmitglieder, Jahresbeitrag $3 \mathrm{M}$. Höhe der Einzelunterstïtzung ca. 40 M., Summe der bisher gezahlten Unterstïtzungen 855 M., Gesammtsumme der bisher der Kasse gewährten Geschenke 3300 M., Zalı der Mitglieder 1898: 42, Vereinsvermögen 6500 M., Zahl der 1897 um Unterstiitzıng Eingekommenen: 2 dringend bediirftige Wittwen).

4. Provinz Schlesien: Unterstützungskasse des Vereins von Aerzten im Riesengebirge (Sitz in Hirschberg).

5. Provinz Sachsen: Unterstützungskasse des Vereins der Aerzte im Regierungsbezirk Merseburg.

6. Provinz Schleswig-Holstein: Wittwenkasse des Aerztlichen Vereins Sonderburg (1892 gegründet; bezieht ihre Einnahmen lediglich ans den Honoraren fïr die Ausstellung von Attesten fïr die Alters- und Invaliditäts-Versicherungsanstalten seitens der Vereinsmitglieder; muterstützt mur bedïrftige Arztwittwen, ansnahmsweise bei vorhandenen Mitteln auch bedürftige Aerzte; Summe der bisher gezahlten Unterstützıngen: ca. 670 M.; Mitgliederzahl 11; Vereinsvermögen 1898: 910 M.; Gesammtunterstützing 1897: $140 \mathrm{M}$. an 3 dringend bediirftige Wittwen und Waisen)

7. Provinz Westfalen: Sterbe-und Unterstïtzungskasse des Aerztlichen Vereins in Hagen (1886 gegrïndet. Beitritt zur Kasse für die Vereinsmitglieder obligatorisch, Jahresbeitrag $10 \mathrm{M}$., Sterbegeld 500 M., Unterstiitzung nach Ermessen des Vorstandes. Gesammtsumme der bisher gezahlten Unterstützungen 5527 M., Gesammtsumme der bisher der Kasse gewährten Geschenke 1454 M., Mitglieder zahl 101, Vereinsvermögen 7280 M., Einnahme 18981150 M., Unterstützung im Jahre 1897 an eine bedürftige Wittwe in Höhe von $200 \mathrm{M}$.).

8. Versicherungs-Unterstiitzungskasse des Aerztlichen Vereins in Hagen (1897 gegründet, hat den Zweck „für die materiellen Schäden und Sorgen, welche ihren Mitgliedern bezw. deren Hinterbliebenen durch Unfälle, in Folge des Alters oder ihres Todes zugefügt

) J. Schwalbe, Die ärztlichen Unterstützungskassen in Deutschland, Dentsche medicinische Wochenschrift 1895 , No. $47 / 48$ 
werden, einzutreten." In Erfüllung dieses Zweckes sollen a) ihre Mitglieder gegen die Folgen von Unfällen jeder Art versichert und b) für das Alter mit einem bezüglich der Höhe und Fälligkeit im Voraus bestimmten Kapital eingekauft werden; auch soll c) für den Fall ihres früheren Todes ihren Hinterbliebenen eine in gleicher Weise fixirte Kapitalsumme garantirt und d) ihnen oder ihren Hinterbliebenen in besonderen Fällen eine periodische oder fortlaufende Subvention gewährt werden. Jedes Mitglied des Hagener ärztlichen Vereins ist eintrittsberechtigt. Die Einnahmen der Kasse bestehen a) in den für die Versicherungszwecke von den Mitgliedern zu erhebenden Beiträgen, b) in den von den rückversichernden Gesellschaften zu gewährenden RabattVergütungen, in Geschenken etc. Jedes Kassenmitglied ist verpflichtet, eine Minimalsumme für seine Versicherung gegen Unfälle, sowie fïr das Alter und im Tode für seine Hinterbliebenen zu zeichnen. Die von der Kasse gewährten Unterstützungen unterliegen dem Ermessen des Vorstandes. Gesammtsumme der bisher gezahlten Unterstützungen 800 M., Mitgliederzahl 50. Vereinsvermögen 11550 M. Gesammteinnahme 18971690 M. Unterstützung im Jahre 1897200 M. an eine Wittwe).

9. Unterstützungskasse des Aerztlichen Vereins des $\mathrm{K}$ reises GeIs en kirchen (unterstützt nur Bedürftige; Vereinsvermögen 1898:3760 M.; hat bisher überhaupt noch keine Unterstützung gezahlt).

10. Rheinprovinz: Wittwen- und Waisenkasse der Aerzte des Saarbrücker Knappschaftsvereins (gegründet 1883; Beitritt nicht obligatorisch; unterstützt alle Berechtigten; Eintrittsgeld $30 \mathrm{M}$. Jahresbeitrag für Verheirathete 90 M., für Unverheirathete 60 M. ; Höhe der Einzelunterstützung an Wittwen und Waisen 200-500 M.; Mitgliederzahl 1898: 47; Vereinsvermögen 58000 M.; Gesammteinnahme 1897 ca. 6000 M.; Gesammtunterstützungen 1897 an 8 Wittwen 3200 M.).

Im Königreich Sachsen, wo das ärztliche Unterstützungs- und Versicherungswesen - wie wiederholt betont werden konnte - bereits seit mehreren Jahren auf hoher Stufe gestanden hat, sind im verflossenen Jahre einige recht bedeutende Erweiterungen erzielt worden. In der Invaliden-Versorgungskasse sind jetzt sämmtliche $28 \mathrm{Be}$ zirksvereine vertreten mit im Ganzen 1760 Mitgliedern. - Die Sterbekasse für die Kreishauptmannschaft Leipzig hat am 22. Angust 1898 neue Satzungen erhalten, aus welchen als wichtigster Punkt hervorzuheben ist, dass bei einem Todesfalle von jedem Mitgliede der Vereine Leipzig-Stadt, Leipzig-Land, Borna und Grimma je $10 \mathrm{M}$. von jedem Mitgliede der Vereine Döbeln, Oschatz und Rochlitz je $6 \mathrm{M}$. als Beitrag erhoben werden und dass dementsprechend für die ersteren Mitglieder das Sterbegeld $1000 \mathrm{M}$. beträgt, während es für die letzteren bei dem bisherigen Satze von 600 M. verbleibt.-Als der wesentlichste Fortschritt ist endlich die Gründung der Wittwen- und Waisenkasse anzusehen, deren Satzungen durch Decret vom 27. October 1898 genehmigt worden sind. Bei der Bedeutung und der vorbildlichen Organisation dieses Instituts lassen wir die wichtigsten Bestimmungen folgen. Die „Wittwen- und Waisenkasse für die ärztlichen Bezirksvereine im Königreich Sachsen “ bezweckt, den von ihren Mitgliedern hinterlassenen Wittwen und Waisen, welche Vermögen oder anderweite Einkünfte in auskömmlichem Maasse nicht besitzen und für ihren Unterhalt nicht oder nicht genügend sorgen können, eine Unterstützung zu gewähren. Der Verein steht unter Aufsicht des Ministeriums des Innern bez. einer von diesem zu bestimmenden Behörde und hat juristische Persönlichkeit. Mitglieder der Wittwen- und Waisenkasse sind die Mitglieder derjenigen ärztlichen Bezirksvereine im Königreich Sachsen, welche der Kasse beigetreten sind (der Beitritt zur Kasse ist also mit anderen Worten fïr die Mitglieder der Bezirksvereine obligatorisch). Ausgenommen vom Beitrittszwang sind nur Assistenten von Krankenanstalten oder praktischen Aerzten, wenn sie keine Privatpraxis treiben dürfen, und ferner pensionsberechtigte, beamtete Aerzte oder active Militärärzte. Der Jahresbeitrag für das einzelne Mitglied beträgt $20 \mathrm{M}$. Bezieht ein Mitglied aus der Invaliden-Versorgungskasse Unterstützung und braucht es keinen Beitrag zu derselben zu zahlen, so ist es während dieser Zeit auch von der Beitragszahlung an die Wittwen- und Waisenkasse befreit. - Die Kasse gewährt den von ihren Mitgliedern hinterlassenen Wittwen und Wrisen im Falle ihrer Bedürftigkeit und auf die Dauer derselben, nach Ablauf der geordneten Wartezeit, Unterstützung in Form einer Rente, welche zunächst für Wittwen $20-50 \mathrm{M}$., für Waisen bis zum vollendeten 18. Lebensjahre 5-25 M. pro Monat betragen soll, deren Höhe aber innerhalb dieser Grenze im einzelnen Falle vom Geschäftsausschusse festgesetzt wird. Die Gewährung der Rente an eine Wittwe hört mit dem Moment der Wiederverheirathung auf. Die Fortgewährung der Rente an Waisen bedarf bei der Wiederverheirathung der Mutter einer erneuten Begutachtung und Bewilligung. Einen Anspruch auf Rente haben Wittwen und Waisen nur dann, wenn 1. der Ehemann bez. Vater vor seinem Tode ununterbrochen zwei Jahre lang Mitglied der Kasse gewesen ist und seine Kassenbeiträge entrichtet worden sind, und 2. nur dann, wenn die Ehe bereits vier Jahre bestanden hatte. Der Geschäftsausschuss ist jedoch ermächtigt, in besonderen Fällen, auch wenn letztere Bedingung nicht erfüllt ist, Unterstützungen zu bewilligen. - Der Anspruch auf Rente kann nicht früher als 3 Monate nach dem Tode des Ehemannes bez. des Vaters oder der Mutter erhoben werden.

Mit dieser Institution haben die sächsischen Aerzte ihrem Gemein- sinn, ihrem Verständuiss für eine wichtige Standesaufgabe und ihrer Thatkraft aufs Neue ein glänzendes Zeugniss ausgestellt. Am 8. Mai 1897 wurde die Gründung einer Wittwen- uud Waisenkasse in der General-Versammlung der Invaliden-Versorgungskasse beschlossen, am 15. Juli 1897 wurde der Satzungsentwurf vom Geschäftsausschuss vorgelegt, und heute ist die Kasse nicht nur ins Leben getreten, sondern sie zählt bereits sämmtliche Bezirksvereine - mit Ausnahme eines einzigen, der indessen seinen Beitritt in Aussicht gestellt hat - zu Mitgliedern. Schon sind der Kasse von Seiten der Collegen Geschenke in der Höhe von ca. $5650 \mathrm{M}$. zugeflossen, theils Beiträge von geselligen Clubs, theils gewonnene Streitobjecte und Bussezahlungen, theils collegiale Honorare für Behandlung von Collegen und deren Familienmitgliedern. Wahrlich, wir anderen Aerzte im Reiche können von dem Genossenschaftsgeist der sächsischen Collegen viel lernen!

Die allgemein anerkannte Ansicht, dass ein gründlich organisirtes Unterstützungswesen an dem erstrebenswerthen festen Zusammenschluss aller Aerzte in erheblichem Grade mitzuwirken geeignet sei, hat in den Verhandlungen der Kreisvereine des Hrossherzogthoms Hessen am 21. April 1898 aufs Neue einen lebhaften Ausdruck gefunden. In der letzten Zeit hat sich unter den hessischen Aerzten das Bedürfniss geltend gemacht, einen die ganze Aerzteschaft des Grossherzogthums umfassenden ärztlichen Landesverein ins Leben zu rufen, dem als Centralorgan die Aufgabe zufallen soll: die wichtigen Fragen, die dem ärztlichen Stand in immer grösserer Zahl und Bedeutung erwachsen, zu bearbeiten, als Mittelpunkt für die Thätigkeit der officiellen Kreisvereine anregend und sammelnd in gemeinschaftlichen Angelegenheiten zu wirken, als Mitglied des deutschen Aerztevereinsbundes die Wahl der Abgeordneten und die Vorberathung der für den deutschen Aerztetag aufgestellten Tagesordnung zu übernehmen u. dgl. m. Den ersten Schritt auf dem Wege, dieses Ziel zu erreichen, erblicken die Kreisvereine in dem Plan, eine ärztliche Landeshilfskasse zu begründen. In der Sitzung der Kreisvereine am 21. April wurde mit überwiegender Majorität beschlossen, eine Hilfskasse für Unterstützung von nothleidenden Aerzten oder deren Hinterbliebenen einzurichten. Mitglieder können alle im ganzen Grossherzogthum Hessen wohnenden Aerzte werden, ebenso wie sich die Unterstützungen auf das ganze Land erstrecken sollen. Zur Ausarbeitung eines Organisationsplans wurde ein Ausschuss von 8 Mitgliedern gewählt. Der Satzungsentwurf, der bereits durchberathen ist, wird, wie mir der Obmann des Ausschusses, Herr Med.Rath Draudt in Darmstadt, freundlichst mittheilt, voranssichtlich im Januar den autorisirten Vertretern der Kreisvereine zur endgiltigen Feststellung vorgelegt werden und damit die ärztliche Hilfskasse ins Leben treten. Die Frage, wie sich die beiden bis jetzt in Hessen bestehenden Unterstützungskassen gegenüber der neu zu gründenden Landeskasse verhalten sollen, wurde zum Theil dadurch erledigt, dass die sogen. Seitz-Stiftung, die bisher lediglich oberhessischen Aerzten zu Gute kam, im Einverständniss mit dem noch lebenden Stifter, Geh. Med.-Rath Prof. Seitz, sich unmittelbar an die neue Institution angliedern soll, während die ausschliesslich für Rheinhessen vorhandene Unterstützungskasse, die über ein Kapital von ca. $50000 \mathrm{M}$. verfügt, durch Aufhebung der ferneren Mitgliederbeiträge als eine feststehende Stiftung, deren Zinsen für rheinhessische Aerzte bezw. deren Hinterbliebene auch weiterhin verwandt werden, mit der Landeskasse vereinigt werden könne. In ähnlicher Weise werden auch kleinere Fonds und Legate, z. B. die Unterstützungskasse des ärztlichen Kreisvereins Dieburg-Erbach, in der neubegrïndeten Landeskasse aufgehen.

Im Herzogthum 0ldenburg ist seit December 1897 der 1853 gegründete Unterstützungsverein nothleidender Aerzte und deren Hinterbliebenen mit dem Aerzte-Verein des Herzogthums Oldenburg verschmolzen, und das Vermögen des ersteren wird als besondere $\mathrm{K}$ asse zur Unterstützung nothleidender Vereinsmitglieder und deren Hinterbliebenen geführt. Nach den neuen Statuten kann, wenn es zu Unterstützungen erforderlich ist, ausser den regelmässigen Veleeinsbeiträgen $(8 \mathrm{M}$. jährlich, von denen $3 \mathrm{M}$. der Unterstützungskasse zufliessen) einmal im Jahre ein Beitrag bis zu 1/3\% des Einkommens aller Art, sei es Rente oder Ertrag der Praxis oder festes Gehalt, soweit es 900 M. übersteigt, von den Mitgliedern erhoben werden. Die Höhe seiner jährlichen Einnahme giebt Jeder nach eigenem besten Ermessen an; es steht jedoch Jedem frei, die Selbstschätzung abzulehmen und die Einschätzung dem Vorstande zu überlassen. Das Vereinsvermögen der Kasse betrug 189810800 M., die Mitgliederzahl 71. Unterstützungen brauchten 1897 nicht gezahlt werden.

Seitens des Vereins der Aerzte im Herzogthum Auhalt haben im Jahre 1898 Verhandlungen über die Begründung einer ärztlichen Unterstützungskasse stattgefunden, die - vie mil Herr Sanitätsrath Günther in Dessau freundlichst mittheilt - bereits zu einem Entwurf, aber noch nicht zur definitiven Organisation einer Kasse geführt haben. Es steht zudem in Aussicht, dass für das Herzogthum eine Aerztekammer ins Leben tritt, der das Besteuerungsrecht verliehen werden soll. Im letzteren Fall werden die schwebenden Fragen in der Angelegenheit der Unterstützungskasse mit einem Schlage gelöst werden.

Im Füstenthmm Lippe besteht ein Unterstützungsverein, der sich auf dio Aerzte des Fürstenthums Lippe und des benachbarten 
preussischen Regierungsbezirks Minden erstreckt. Sein Zweck ist, Mitglieder, welche in Folge von Alter, Krankheit oder Unglücksfällen zeitweilig oder dauernd unterstützungsbedürftig sind, sowie deren Wittwen und Waisen unter den gleichen Voraussetzungen, eine einmalige oder jährliche Beihülfe zu sichern. Die Höhe des Eintritts gelds beträgt, solange der Kapitalfonds $5000 \mathrm{M}$. nicht erreicht hat, bis zum vollendeten 35 . Jahre $5 \mathrm{M}$., bis zum vollendeten 40 . Jahre $10 \mathrm{M}$., bis zum 45. Jahre $15 \mathrm{M}$., bis zum 50 . Jahre $20 \mathrm{M}$., bei mehr als 50 Jahren $30 \mathrm{M}$. Der jährliche Beitrag beträgt $10 \mathrm{M}$. Ausserdem zallen die Mitglieder beim Tode eines Genossen je 5 M. Berechtigung zu einer einmaligen oder jührlichen Unterstïtzung hat jedes Mitglied, welches 3 Jahre hindurch den jährlichen Beitrag bezahlt hat. Beim Todesfalle eines Mitglieds erhält dessen Wittwe oder Erben die von den überlebenden Mitgliedern zu zahlenden $5 \mathrm{M}$. sofort eingeschickt, auch wenn dasselbe dem Verein noch nicht 3 Jahre lang angehört hat (also anscheinend ohne Prüfung der Bedürftigkeit). Das Anrecht zum Bezıng der Unterstïtzungen erlischt, wenn die Wittwe eines Mitglieds eine neue Ehe eingeht, bezw. wenn die Kinder das 18. Lebensjahr erreicht haben.

Neuerdings ist für das Fürstenthum Lippe eine Standesordnung geplant, in deren Rahmen eine Pensions- und Wittwenkasse mit obligatorischem Beitritt sämmtlicher Lippischen Aerzte Platz finden soll. Die Statuten dieser Kasse sind - nach einer Auskunft des Herrn Medicinalrath $\mathrm{O}$ verbeck in Lemgo - bereits ausgearbeitet, doch unterliegen sie noch der Prüfung und Bestätigung seitens der Regierung.

In der Stadt Iübeck hat der dortige ärztliche Verein 1898 eine Sterbekasse gegrïndet. Der Beitritt ist für die Mitglieder des Vereins obligatorisch. Der Jahresbeitrag beträgt 20 M., das Sterbegeld für die Hinterbliebenen $1000 \mathrm{M}$. Mitgliederzahl 54, Vereinsvermögen $1540 \mathrm{M}$.

So viel über den letztjährigen Ausbau des deutschen ärztlichen Unterstützungs- und Versicherungswesens. Mancher erhebliche Fortschritt ist zu verzeichnen, mehr aber bleibt noch zu thun übrig, um das Ziel zu erreichen, das in der 2. These der Denkschrift unserer Aerztekammer bezeichnet ist als: möglichste Vorbeugung von Nothfällen und im Falle eintretender Noth standescemässe Hülfe für jedweden Standesgenossen und dessen Familie. 